\title{
El fenómeno de la caída libre en Galileo
}

\author{
The PHenomenon of the fRee fall in Galileo
}

\author{
María Lilia Perilla Perilla*
}

\begin{abstract}
Resumen
El primero en formular la hipótesis de que todos los cuerpos caen con la misma velocidad en el vacío, independientemente de su peso, fue Galileo, por las siguientes razones experimentales: cuanto menor es la densidad del medio, tanto menor es la ventaja de un cuerpo sobre el otro al caer desde una misma altura, por consiguiente, en el vacío, cuya densidad es nula, la ventaja es nula, es decir, todos los cuerpos caen con la misma velocidad independientemente de sus pesos absolutos. Sin embargo, a pesar de que la argumentación es irreprochable y está respaldada por innumerables experimentos supremamente ingeniosos, el genio de Galileo no logra resolver satisfactoriamente la objeción aristotélica de que debe haber proporción entre la causa y el efecto. Si la causa del movimiento es el peso, entonces, a mayor peso, mayor movimiento, si se elimina toda resistencia. La solución definitiva sólo es posible a partir de Newton, con la distinción entre masa gravitacional y masa inercial.
\end{abstract}

Palabras clave: Caída libre, Galileo, peso, velocidad, distancia.

\begin{abstract}
The first one in formulating the hypothesis that all the bodies fall with the same speed in the vacuum, independently of its weight, it was Galileo, for the following experimental reasons: whichever minor is the density of the means, as much smaller is the advantage of a body on the other when falling from a same height, consequently, in the vacum, one whose density is null, the advantage is null, that is to say, all the bodies fall independently with the same speed of their absolute weights. Nevertheless, although the argumentation is irreproachable by innumerable supremely ingenious experiments, the genius of Galileo does not manage satisfactorily to solve the aristotelian objection which it must have proportion between the cause and the effect. If the cause of the movement
\end{abstract}

\footnotetext{
* Universidad de la Sabana, Facultad de Ingeniería, Área de Matemáticas Aplicadas y Estadistica, maliper@andinet.com
} maria.perilla@unisabana.edu.co 
is the weight, then, to greater weight, greater movement, if any resistance is eliminated.

The definitive solution only is possible from Newton, with the distinction between gravitational mass and inertial mass.

Key words: Free fall, Galileo, weight, speed, distance.

\section{INTRODUCCIÓN}

El presente artículo es un extracto parcial de un capítulo de la tesis doctoral El fenómeno de la caída libre y los conceptos de masa gravitacional y masa inercial, presentada al Programa de Doctorado de la Universidad Pedagógica Nacional. La elaboración de la tesis se llevó a cabo dentro de la línea de investigación la elaboración de los conceptos científicos, dirigida por el doctor Fabio Vélez U.

El primero de los filósofos antiguos en considerar, así fuera brevemente, el fenómeno de la caída libre, fue Aristóteles. La discusión se enmarca dentro del principio filosófico de que el efecto debe ser proporcional a la causa. Ahora bien, si la causa es el peso y si el efecto es la velocidad de caída, entonces, no hay más remedio que postular que los cuerpos más pesados caen más rápido. Pero el peso no es la única causa, el medio ofrece resistencia, en consecuencia, la velocidad de caída es proporcional al peso e inversamente proporcional a la resistencia. En un mismo medio, sin embargo, la resistencia del medio es la misma para todos los cuerpos, por consiguiente, según Aristóteles, en el aire no sólo los cuerpos más pesados caen más rápido, sino que un cuerpo 10 veces más pesado que otro debería caer 10 veces más rápido. Por extraño que pueda parecer, esta aseveración permaneció sin cuestionarse por más de 15 siglos. Uno de los primeros pensadores en criticar la posición aristotélica fue Juan Bautista Benedetti, maestro de Galileo. Dejando a un lado las especulaciones filosóficas y tomando como base razonamientos físicos y matemáticos, el sabio italiano llega a sostener la tesis de que todos los cuerpos del mismo peso específico caen con la misma velocidad en el vacío, independientemente de sus pesos absolutos: una gota de plomo cae con la misma velocidad que una bala de mosquete, 1.000 veces más pesada. Galileo, por razones experimentales, da un paso más allá: en el vacío, todos los cuerpos, independientemente de su peso específico y absoluto, caen con la misma velocidad. Más aún, llega a proponer una fórmula matemática, completamente artificial, que explicaría no sólo la igualdad de velocidad en el vacío, sino la poca diferencia entre las velocidades de caída en un medio, como el aire, de cuerpos de pesos absolutos notoriamente diferentes. Sin embargo, el gran genio de Galileo no encuentra una explicación teórica, no puramente aritmética, a la igualdad de velocidades, a pesar de la diferencia de pesos: si en el vacío no hay resistencia, ¿cómo es posible que el cuerpo más pesado no caiga más rápido? En el fondo, Galileo no sabe cómo conciliar su descubrimiento con la tesis aristotélica de que 
debe existir cierta proporcionalidad entre el efecto y la causa.

Aunque Newton no se ocupa directamente del fenómeno de la caída libre, por estar ocupado en problemas de mucha mayor envergadura, sus principios físicos proporcionan los elementos necesarios para una solución definitiva, logrando lo que nadie más había logrado en los 17 siglos anteriores. El peso es una fuerza exterior ejercida sobre el cuerpo por la Tierra. En todo movimiento intervienen dos causas, para hablar en términos aristotélicos: la fuerza atractiva, que es el peso, y la resistencia a ser movido, que es la inercia, cuya medida se denomina masa inercial. Por una extraña circunstancia, que la física aún no ha podido explicar, a mayor fuerza atractiva, mayor resistencia, es decir, mayor masa inercial. El aforismo aristotélico es cierto si el efecto depende de una sola causa, pero en el caso de la caída libre depende de dos causas, la atractiva y la resistiva. La relación de las dos causas se expresa analíticamente en la famosa segunda ley, según la cual la aceleración es directamente proporcional a la fuerza e inversamente proporcional a la masa inercial.

\section{GaliLeo}

\section{La hipótesis de Juan Bautista Benedetti}

El físico italiano más importante del siglo $X V I$, según Alexander Koyré, influyó de manera notable sobre el joven Galileo. Entre sus obras físicas y matemáticas se encuentran dos tratados, Resolución de todos los problemas de Euclides y el Libro de diversas consideraciones matemáticas y físicas, publicado en 1585. Por razones hidrostáticas, que no es del caso explicar en este momento, Benedetti llegó a la conclusión de que la velocidad de caída dependía del peso específico (peso relativo) y no del peso absoluto. Los cuerpos del mismo peso específico caían con la misma velocidad, independientemente de sus pesos absolutos. Una gota de plomo caía con la misma velocidad que una bala de cañón, también de plomo. Sin embargo, cuando se compara la velocidad de caída de cuerpos de diferente peso específico, los de mayor peso específico caen con mayor velocidad, independientemente de sus pesos absolutos. Es decir, de acuerdo con esta segunda hipótesis, una gota de oro cae más rápido que una bala de cañón, de plomo, aunque ésta pudiera pesar cien o mil veces más que la otra.

\section{La argumentación experimental}

Si se tiene en cuenta que la fuerza motriz es precisamente el peso, parece verosímil pensar que las velocidades de caída en un mismo medio son directamente proporcionales a los respectivos pesos, como propone Aristóteles. Pero la experiencia controlada y la misma experiencia ordinaria parecen contradecir abiertamente la tesis aristotélica. Si Aristóteles tuviera razón, una piedra de 100 libras caería 10 veces más rápido que una piedra de 1 libra ${ }^{1}$, lo que significaría que ésta

\footnotetext{
l Se trata evidentemente de una rapidez media, no de una rapidez instantánea. Dentro de este contexto, un cuerpo cae más rápido que otro si recorre la misma distancia en menor tiempo.
} 
sólo habría recorrido 10 brazas cuando la otra hubiera recorrido 100 brazas, en abierta oposición con la experiencia cotidiana que nos dice que la ventaja que le tomaría la más pesada a la menos pesada es mucho menor, quizás de algunos dedos. El famoso experimento de Pisa, en el que supuestamente Galileo había dejado caer cuerpos de diferentes sustancias y de diferentes pesos desde la altura de la torre, parece confirmar la proposición de que la diferencia de recorrido es casi imperceptible y que, por consiguiente, en el aire, las velocidades de todos los cuerpos es casi la misma.

Aunque Galileo no menciona a Benedetti en ningún momento, la crítica que hace a Aristóteles también se aplica a éste, pues de acuerdo con Benedetti, la diferencia de velocidades entre la madera y el plomo, por ejemplo, sería de 5,5, si se supone que el aire es mil veces menos denso que el agua, como se mostró en los ejemplos expuestos anteriormente. Pero la experiencia, nuevamente, no parece mostrar que el plomo le tome una ventaja tan grande a la madera. Es cierto que Benedetti afirma que todos los cuerpos de igual peso específico caen con la misma velocidad en el mismo medio, y en esto está mucho más conforme con la experiencia cotidiana, pues si suponemos que las piedras tienen el mismo peso específico o un peso específico muy semejante, entonces sus velocidades de caída son iguales o muy similares. Galileo es de la misma opinión: los cuerpos homogéneos, de la misma forma y de pesos específicos iguales, caen con la misma velocidad. Pero el problema, piensa Galileo, no es el de la caída de los cuerpos de pesos específicos iguales, sino los de pesos específicos distintos, como el de una vejiga llena de aire y un cuerpo de plomo del mismo volumen que puede pesar mil veces más. La experiencia muestra que, en este caso, la ventaja de la esfera de plomo no será el triple, ni siquiera el doble, aunque según Aristóteles debería ser mil veces mayor ${ }^{2}$.

Es posible que si la altura de caída es bastante grande, la diferencia de las velocidades sea notoria, como lo sugiere Simplicio con motivo del experimento mental de la vejiga y la gota de plomo. Salviati acepta la objeción de Simplicio, pero al mismo tiempo hace ver que si la ventaja de un cuerpo sobre otro varía con la altura, no es a causa del mayor o menor peso, sino de la resistencia del medio que se va haciendo mayor a medida que aumenta la velocidad del cuerpo y que se hace más notoria en el cuerpo menos pesado que en el más pesado, de tal manera que se puede suponer que en el vacío, en donde el medio no ofrece ninguna resistencia, los cuerpos caen con la misma velocidad, independientemente de sus pesos y de la mayor o menor altura desde la que inician su movimiento de caída ${ }^{3}$.

Si la resistencia del medio se hace más notoria en alturas de caída relativamente grandes, a causa del aumento de

\footnotetext{
2 El experimento de la vejiga y de un cuerpo de plomo del mismo volumen se encuentra en Consideraciones y demostraciones matemáticas sobre dos nuevas ciencias. Galileo, (1976), p. 163.

3 Ibíd, pp. 162-163.
} 
la resistencia del medio con el cuadrado de la velocidad del cuerpo, entonces, concluye Galileo, los experimentos se deben realizar con alturas pequeñas, como, por ejemplo, los llevados a cabo en planos inclinados. Aunque no hay una relación pormenorizada de los experimentos realizados, parece que fueron muchos y variados. Ahora bien, en todos los casos la ventaja de unos cuerpos sobre otros era imperceptible, a pesar de que la diferencia de pesos era notable y de que los cuerpos eran de sustancias tan disímiles como la madera, el oro y el plomo. Las ventajas eran inapreciables, es cierto, pero ¿no se debería precisamente esta pequeñez de las ventajas a la pequeñez relativa de las alturas de los planos inclinados? Se presentaba entonces un dilema, si las alturas eran grandes, la resistencia del medio distorsionaba los resultados; si las alturas eran pequeñas, como sucedía con los planos inclinados, los resultados no eran suficientemente concluyentes. Por eso había que buscar otro tipo de experimento en donde se pudieran llevar a cabo sucesivamente caídas pequeñas, de tal manera que el efecto de todas ellas se pudiera acumular. El genio experimental de Galileo encontró prontamente la solución. El movimiento de vaivén de un péndulo equivale a un número determinado de caídas pequeñas, consecutivas. Si se dejan oscilar libremente dos esferas de diferente peso y si después de un número relativamente alto de oscilaciones uno de los cuerpos no le coge ventaja apreciable al otro, entonces se puede concluir que los dos cuerpos caen prácticamente con la misma velocidad, en el aire.
La tesis de que los cuerpos caen con la misma velocidad en el vacío, independientemente de sus pesos, es tan nueva y atrevida que Simplicio y Sagredo no dudan en expresar su asombro. Pero las razones y experimentos propuestos por Salviati son tan convincentes que se declaran satisfechos.

Las razones y experimentos propuestos parecen confirmar la hipótesis de que en el aire los cuerpos caen con velocidades muy cercanas. Pero, ¿cómo se concluye de aquí que las velocidades en el vacío son completamente iguales? El argumento es supremamente convincente y original. A medida que disminuye la densidad del medio, disminuye la diferencia de velocidades o la ventaja que un cuerpo le saca al otro. En el mercurio, el oro le saca una ventaja enorme al plomo, si se tiene en cuenta que es el oro el que cae, mientras que el plomo flota. En el agua, ambos cuerpos caen, pero el otro le saca una ventaja considerable. En el aire la diferencia es insignificante, por consiguiente, en el vacío, la ventaja debe ser nula. En consecuencia, las velocidades de caída en el vacío son perfectamente iguales.

\section{La argumentación a priori}

Aunque Galileo está completamente satisfecho con la demostración experimental de que todos los cuerpos caen con la misma velocidad en el vacío, quiere dar un paso más allá de lo puramente experimental, y recurre para esto a una demostración que curiosamente ha pasado desapercibida para sus contemporáneos y para los comentaristas posteriores. El 
lector, como dice Simplicio en el diálogo pertinente, queda ofuscado sin saber qué decir, pues a pesar de que parece una argumentación sofística, sin embargo, no es fácil encontrar el error lógico, ya que desde el punto de vista teórico ni Galileo, ni ninguno de sus contemporáneos estaban en capacidad de dar una solución adecuada. Un argumento semejante se encuentra en Benedetti, de quien Galileo sacó al menos la idea general. El argumento es el siguiente. Si los cuerpos más pesados caen más rápido, entonces dos cuerpos amarrados por una cuerda, deben caer más rápido y menos rápido que el mayor de ellos. Más rápido porque los dos juntos pesan más que el mayor, y menos rápido porque el menor, que cae más lentamente, retrasa el movimiento del mayor. A primera vista, la solución parece fácil, basta con negar que dos cuerpos unidos por una cuerda, mientras caen, pesan más que uno solo de ellos. En realidad los cuerpos, mientras caen, no pesan, Galileo lo sabe bien, como aparece en el diálogo ${ }^{4}$. Pero es evidente que cuando se afirma que los cuerpos más pesados caen más rápido, no se refiere al peso mientras caen, sino al peso, antes de soltarlos, y entonces no se puede negar que dos cuerpos, unidos por una cuerda, tan corta como se quiera, es decir, dos cuerpos juntos, pesan más que uno solo de ellos. Para hacer más agudo el dilema, supóngase una esfera de plomo hueca que tiene en su interior otra esfera también de plomo, que llena

4 Consideraciones y demostraciones matemáticas sobre dos nuevas ciencias, Galileo, (1976), p. 149. todo el espacio vacío de la primera. $\mathrm{Si}$ los cuerpos más pesados caen más rápido, entonces parece evidente que las dos esferas juntas pesan más que la mayor de ellas y por consiguiente deberían caer más rápido; pero al mismo tiempo, la esfera interior, que es menos pesada que la esfera hueca, le debe restar al sistema formado por las dos esferas. En consecuencia, las dos esferas juntas caen más rápido y menos rápido que la esfera hueca. ¿Cómo se resuelve el dilema? De acuerdo con la conclusión de la discusión entre Simplicio y Salviati, en el diálogo mencionado, no hay más remedio que negar la suposición que los cuerpos más pesados caen más rápido, si tienen el mismo peso específico. Concluyamos, dice Galileo en boca de Salviati, que los móviles, grandes o pequeños, se mueven con la misma velocidad si tienen el mismo peso específico ${ }^{5}$.

A continuación transcribimos la parte fundamental del diálogo, tal como aparece en el tratado de Galileo, que lleva el nombre de Consideraciones y demostraciones sobre dos nuevas ciencias.

Simplicio: Está fuera de toda duda que el mismo móvil en el mismo medio tiene una velocidad reglamentada y determinada por la naturaleza, la cual no podrá aumentarse a no ser por un impulso nuevo ni disminuirse si no es recurriendo a algo que la obstaculice y la retarde.

Salviati: Entonces, si nosotros tuviéramos dos móviles, cuyas velocidades naturales fuesen distintas, es evidente que si unié-

\footnotetext{
5 Ibid, p. 151
} 
semos ambos, el más rápido perdería velocidad por obra del más lento, mientras que éste aceleraría debido al más rápido. ¿Estáis de acuerdo con lo que acabo de decir?

Simplicio: Me parece que las cosas deben, ciertamente, suceder así.

Salviati: Pero si esto es así, y si es verdad, por otro lado, que una piedra grande se mueve, por ejemplo, con una velocidad de ocho grados y una piedra pequeña, con una velocidad de cuatro, si las unimos, el resultado de ambas, según lo dicho, será inferior a ocho grados de velocidad, Ahora bien, las dos piedras juntas dan por resultado una más grande que la primera que se movía con ocho grados de velocidad; de lo que se sigue que tal compuesto se moverá a más velocidad que la primera de las piedras sola, lo cual contradice vuestra hipótesis. Veis, pues, cómo suponiendo que el móvil más pesado se mueve a más velocidad que el que pesa menos, concluyo que el más pesado se mueve a menos velocidad.

Si el argumento fuera correcto, habría que concluir que es imposible imaginar un mundo donde los cuerpos más pesados caigan más rápido, lo que no se puede admitir de acuerdo con la física newtoniana, en donde se diferencia la masa inercial de la masa gravitacional. ¿En dónde está, pues, el error lógico de Galileo, ya que el error teórico supone conceptos posteriores que Galileo no está obligado a conocer y mucho menos a aplicar?

El argumento, por una parte, considera los dos cuerpos juntos como un solo cuerpo más pesado que cualquiera de ellos, y por otra parte, como dos cuerpos, y todo esto bajo un mismo aspecto, el de la velocidad de caída. Evidentemente, desde el punto del equilibrio estático, dos piedras se pueden reemplazar por una sola piedra cuyo peso resulte de sumar los dos pesos individuales y cuyo centro de gravedad coincida con el centro de gravedad del sistema formado por las dos piedras. Pero lo que sucede desde el punto de vista del equilibrio estático no tiene que suceder, sin más, desde el punto de vista cinético. Si se pueden reemplazar, cinéticamente, por una sola piedra, en las condicionadas mencionadas, entonces la velocidad del centro de gravedad del sistema formado por las dos piedras sería la misma que la del centro de gravedad de la piedra única. Si no se pueden reemplazar las dos piedras juntas por una sola, entonces la velocidad del centro de gravedad del sistema no tiene por qué ser igual a la velocidad del centro de gravedad de la piedra única. El error de Galileo, pues, es el mismo de Benedetti, el inferir de una equivalencia estática una equivalencia cinética, sin demostración de ninguna clase. Pero el lector comprenderá que estas reflexiones estaban fuera del alcance de Galileo y de sus contemporáneos. Esto explica, posiblemente, que ninguno de sus contemporáneos se haya tomado la molestia de analizar un argumento tan convincente y que, en palabras de Simplicio, lo deja a uno completamente ofuscado, "pues me parece que la piedra más pequeña unida a la mayor le da más peso, y no consigo explicarme cómo dándole más peso, no deba sumarle velocidad o, al menos, no disminuírsela". 
El argumento de Galileo se puede exponer de una manera más formal de la siguiente manera. Supóngase que $A$ y $B$ son del mismo tamaño, de la misma forma y de la misma sustancia. Supóngase que los cuerpos $\mathrm{B}$ y $\mathrm{C}$, unidos por una barra, de masa despreciable, se pueden reemplazar, desde el punto de vista del equilibrio estático, por el cuerpo $D$, cuyo peso es igual a la suma de los dos pesos individuales $B$ y $C$. Supóngase además que e es el centro de gravedad del sistema formado por los cuerpos B y C.

Ahora bien, el centro de gravedad del sistema formado por B y C cae con la misma velocidad que el centro de gravedad del cuerpo $D$, ya que sus pesos son iguales. El cuerpo $D$ cae más rápido que el cuerpo A, porque es más pesado (grande), por tanto el sistema $B C$, que cae con la misma velocidad que el cuerpo $D$, cae más rápido que el cuerpo $A$. Pero el sistema $B C$ no puede caer más rápido que el cuerpo $A$, porque $C$ le resta velocidad al cuerpo $B$, que es igual a A. Por consiguiente, si se acepta la hipótesis de que los cuerpos más pesados caen más rápido se seguiría la contradicción de que un mismo cuerpo cae más rápido y menos rápido que otro cuerpo. Por tanto, la hipótesis es falsa y todos los cuerpos caen con la misma velocidad, al menos, si tienen el mismo peso específico.

El lector habrá notado que del hecho de que $B$ y $C$ unidos caigan más rápido que $A$, porque pesan más, se infiere arbitrariamente que por ese solo hecho, mientras $B$ permanece unido con $C$ también cae más rápido que $\mathrm{A}$, lo que habría

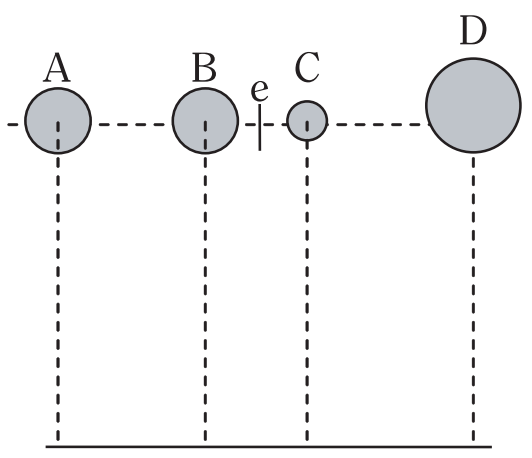

que demostrar. La confusión se origina en el hecho de que no se especifica cómo se determina la velocidad de caída de dos cuerpos cuando están unidos. Parece evidente, por el contexto, que esa velocidad se determina por la velocidad del centro de gravedad de los dos cuerpos. Si es así, bien puede suceder que el centro de gravedad del sistema caiga más rápido que $A$ y que, sin embargo, $B$, que es igual a $A$, caiga con la misma velocidad que éste, como se puede ver claramente en la figura adjunta. El pensamiento implícito en la argumentación es que los cuerpos B y $C$ se pueden reemplazar por el cuerpo $D$, desde el punto de vista del peso. Pero entonces el argumento supondría, por una parte, que los dos cuerpos juntos se pueden reemplazar por un solo cuerpo y, por la otra, que no se pueden reemplazar por un solo cuerpo, sino que se comportan en la caída como dos cuerpos independientes, de tal manera que el cuerpo $C$ le resta algo de velocidad al cuerpo B. 


\section{LA FÓRMULA DE LA CAÍDA LIBRE}

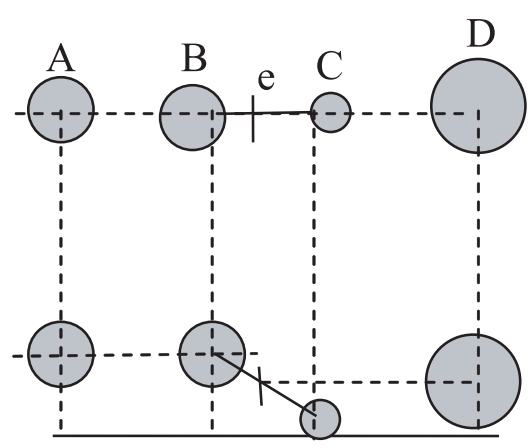

La velocidad del sistema $B C$ es la misma del cuerpo $D$, sin embargo, de ahí no se sigue que el cuerpo $\mathrm{B}$ caiga más rápido que el cuerpo $\mathrm{A}$.

Los experimentos convencieron a Galileo de que todos los cuerpos, independientemente de sus pesos absolutos, caen con la misma velocidad en el vacío. Pero Galileo carece de una teoría adecuada para justificar su tesis, ni siquiera puede recurrir a las consideraciones hidrostáticas de Benedetti, que sólo alcanzan a demostrar que los cuerpos de pesos específicos iguales caen con la misma velocidad en un mismo medio. En ausencia de una teoría, se ve forzado a construir una ecuación completamente artificial que concuerda, dentro de cierto grado de aproximación, con los resultados experimentales y con la suposición de que todos los cuerpos caen con la misma velocidad en el vacío. Los resultados experimentales muestran que la ventaja que un cuerpo le toma a otro en un mismo medio es relativamente pequeña a pesar de la gran diferencia de pesos. La fórmula de Benedetti no es suficiente, piensa Galileo, pues mientras que establece que un cuerpo de plomo cae 5,5 veces más rápido que la madera en el aire, independientemente del peso, la experiencia muestra que la diferencia es mucho menor, de algunos dedos en el mejor de los casos. El segundo tipo de resultados experimentales muestra que a medida que se reduce la densidad del medio, disminuye la ventaja que un cuerpo le coge a otro, independientemente del peso. La velocidad por lo tanto, en un medio cualquiera, es proporcional, piensa Galileo, a la fracción que resulta de quitarle a la unidad el inverso del peso específico del cuerpo. Si se llama $c$ a la velocidad del cuerpo en el vacío, entonces, la velocidad de ese cuerpo en un medio dado es el producto de $c$ y de $(1-1 / \gamma)$, en donde $\gamma$ es el peso específico del cuerpo con respecto al medio en el que cae el cuerpo. Si se expresa algebraicamente la velocidad de un cuerpo en un medio dado, entonces la velocidad estaría dada por la ecuación: $v=c(1-1 / \gamma)$, lo que $c$ es la velocidad en el vacío, que se supone igual para todos los cuerpos, y $\gamma$ el peso relativo del cuerpo con respecto al medio. Todo sucede como si el medio disminuyera la velocidad del móvil en el vacío en una proporción que está dada por $1 / \gamma$. Dentro de este contexto, se entiende la formulación de Galileo: Y como es evidente que el medio aligera los cuerpos inmersos en él, en proporción al peso de un volumen igual de su propia materia, obtendremos lo que buscamos (la velocidad en un medio dado) si disminuimos, en la misma proporción, la velocidad de los móviles que en el medio no resistente (tal y como hemos supuesto) serían iguales ${ }^{6}$.

$6 \quad$ Ibid, art. 121, p. 166. 
Si se expresa la fórmula por medio de una proporción, entonces se puede indicar de la siguiente manera:

$$
\frac{v_{A 1}}{v_{B 2}}=\frac{\left(1-\frac{1}{\gamma_{A 1}}\right)}{\left(1-\frac{1}{\gamma_{B 2}}\right)}
$$

en donde $v_{A 1}$ significa la velocidad del cuerpo A en el medio (1) y $\gamma_{A 1}$ significa el peso específico del cuerpo $A$ con respecto al medio (1).

Evidentemente, la fórmula de Galileo no tiene ninguna importancia física. Confirma, eso sí, la hipótesis de que Galileo no tiene ningún sistema teórico para justificar la famosa hipótesis de que todos los cuerpos caen con la misma velocidad en el vacío, independientemente de sus masas.

\section{Conclusión}

Por medio de una extrapolación supremamente convincente y audaz, Galileo formula la hipótesis de que todos los cuerpos caen con la misma velocidad en el vacío, independientemente de su masa. Sin embargo, queda una dificultad teórica que no logró resolver a pesar de ingentes esfuerzos. Si la velocidad de caída es un efecto del peso, ¿por qué los cuerpos más pesados no caen más rápido en el vacío? Desde el punto de vista teórico sólo hay una respuesta posible: la velocidad de caída no es efecto solamente del peso, sino de otra causa desconocida: la masa inercial. Los cuerpos más grandes, para utilizar la expresión de Galileo, no solamente son más pesados, sino que también oponen más resistencia al movimiento. Kepler fue el primero en sospechar que había una cierta resistencia al movimiento, pero Newton fue quien resolvió definitivamente el problema de la caída libre.

\section{Bibliografía}

Drake, S. (1970). Galileo studies. The University of Michigan: Ann Arbor.

Galileo, G. (1993). Diálogos sobre los dos máximos sistemas del mundo ptolemaico y copernicano. Madrid: Alianza.

Galileo, G. (1976). Consideraciones y demostraciones sobre dos nuevas Ciencias. Madrid: Editora Nacional.

Jammer, M. 1997. Concepts of mass. New York: Dovers Publications.

Kant, E. (1983). Principios metafísicos de la ciencia de la naturaleza. Madrid: Alianza.

Koyré, A. (1978). Estudios de historia del pensamiento científico. México: Siglo XXI.

Koyré, A. (1981). Estudios Galileanos. México: Siglo XXI.

ARTíCULO RECIBIDO: 1-06-2005

Y APROBADO: 11-11-2005 